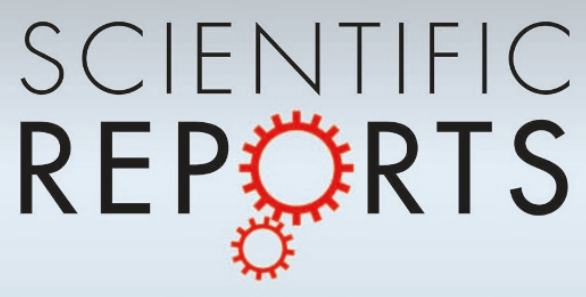

open Continuous-flow Mass Production of

SUBJECT AREAS: Silicon Nanowires via

NANOWIRES

SYNTHESIS AND PROCESSING

Received

5 September 2013

Accepted

3 December 2013

Published

13 January 2014

\title{
Substrate-Enhanced Metal-Catalyzed Electroless Etching of Silicon with Dissolved Oxygen as an Oxidant
}

\author{
Ya Hu' ${ }^{1}$ Kui-Qing Peng' , Lin Liu' , Zhen Qiao', Xing Huang ${ }^{2}$, Xiao-Ling Wu' , Xiang-Min Meng ${ }^{2}$ \\ \& Shuit-Tong Lee ${ }^{3}$
}

Correspondence and requests for materials should be addressed to

K.-Q.P. (kq_peng@ bnu.edu.cn) or S.-T.L. lapannale@suda.edu.

\footnotetext{
'Department of Physics and Beijing Key Laboratory of Energy Conversion and Storage Materials, Beijing Normal University, Beijing, China, ${ }^{2}$ Technical Institute of Physics and Chemistry, Key Laboratory of Photochemical Conversion and Optoelectronic Materials, Chinese Academy of Sciences, Beijing, China, ${ }^{3}$ Institute of Functional Nano and Soft Materials (FUNSOM) and Collaborative Innovation Center of Suzhou Nano Science and Technology, Soochow University, Suzhou, China.
}

Silicon nanowires (SiNWs) are attracting growing interest due to their unique properties and promising applications in photovoltaic devices, thermoelectric devices, lithium-ion batteries, and biotechnology. Low-cost mass production of SiNWs is essential for SiNWs-based nanotechnology commercialization. However, economic, controlled large-scale production of SiNWs remains challenging and rarely attainable. Here, we demonstrate a facile strategy capable of low-cost, continuous-flow mass production of SiNWs on an industrial scale. The strategy relies on substrate-enhanced metal-catalyzed electroless etching (MCEE) of silicon using dissolved oxygen in aqueous hydrofluoric acid (HF) solution as an oxidant. The distinct advantages of this novel MCEE approach, such as simplicity, scalability and flexibility, make it an attractive alternative to conventional MCEE methods.

arge-scale fabrication of $\mathrm{SiNWs}^{1-7}$ is of great importance in pursuit of exploring SiNWs for wide-ranging technological applications ${ }^{8-16}$ in electronics, photonics, renewable energy, and biotechnology. Despite intense efforts, mass production of SiNWs on an industrial scale remains unattainable. SiNWs are commonly produced by chemical vapor deposition methods ${ }^{7}$ via vapor-liquid-solid (VLS) mechanism. However the VLS method can only produce SiNWs in limited quantities. Recently, metal-catalyzed electroless etching (MCEE) of silicon $^{17-30}$, also known as metal-assisted chemical etching (MACE), has been widely used to fabricate high-aspect ratio SiNWs and other silicon nanostructures such as nanoholes due to its simplicity and low cost. The nature of MCEE of silicon in aqueous HF solution is galvanic corrosion. In traditional well-established MCEE methods, common oxidizing agent such as hydrogen peroxide or silver nitrate is indispensable ${ }^{17-30}$. To date, MCEE SiNWs have been widely investigated for photovoltaic device, thermoelectric device, lithium-ion battery, and biological applications ${ }^{9,31-37}$.

Here we report simple and low-cost MCEE of silicon in aerated aqueous HF solution enabling mass production of SiNWs with unprecedented ease. The process relies on substrate-enhanced MCEE of silicon using dissolved oxygen in aqueous HF solution as a green oxidant, which is ubiquitous in nature, yet completely neglected in traditional MCEE methods due to sluggish oxygen reduction kinetics. We demonstrate that this approach can establish continuous-flow production of SiNWs on an industrial scale, offering unparalleled advantages over traditional approaches in terms of cost, processing time, and versatility. The continuous-flow mass-production process for SiNWs is schematically shown in Fig. 1 (see Methods for details). The production line consists of conductive graphite (or noble metal such as $\mathrm{Ag}, \mathrm{Au}$, and $\mathrm{Pt}$ ) substrates immersed in aerated aqueous HF solution, in which SiNW arrays are produced by continuously flowing Si substrates through the aerated aqueous HF solution. 


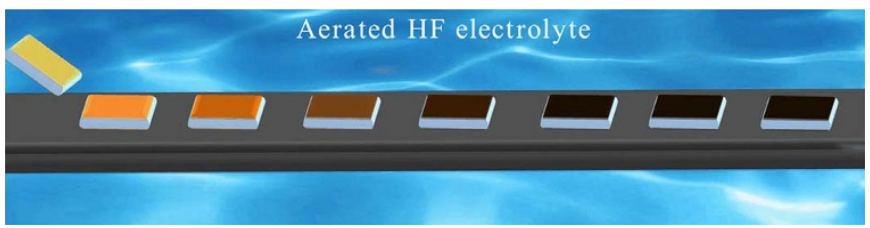

Figure $1 \mid$ Schematic overview of continuous production process for SiNWs via substrate-enhanced MCEE of silicon in aerated aqueous HF solution. Silicon substrates coated with noble metal particles are placed in close contact with graphite substrates, and silicon nanowires are produced after passing silicon substrates once through the aerated aqueous HF solution.

\section{Results}

Graphite substrate-enhanced MCEE of silicon in aerated aqueous HF solution. To promote selective Si corrosion and create highaspect ratio SiNWs, the surfaces of the starting Si materials must be pre-patterned with noble metal catalysts such as silver or gold (silver nanoparticles, AgNPs are used here). Otherwise, only a thin porous $\mathrm{Si}$ layer would be formed from bare Si wafer or powders (Fig. S1). Our experimental results show that aligned SiNWs could be created on Si wafers of different doping types and concentrations. Figure $2 \mathrm{a}$ and $2 \mathrm{~b}$ show respectively the typical cross-sectional scanning electron microscope (SEM) images of large-area vertically aligned SiNW arrays prepared on p-type $1-10 \Omega \cdot \mathrm{cm} \mathrm{Si}(100)$ and ntype $2-3 \Omega \cdot \mathrm{cm} \mathrm{Si}(100)$ wafers after one pass (2 hours) through aerated aqueous HF solution. The SEM images clearly show that the corrosion rate of $\mathrm{p}-\mathrm{Si}$ is faster than that of $\mathrm{n}-\mathrm{Si}$ under the same conditions, revealing different corrosion behaviors of $\mathrm{p}$ - and n-type silicon due to their distinct charge carrier characteristics. The asprepared SiNWs are typically $20-250 \mathrm{~nm}$ in diameter, while wire lengths could be simply controlled by changing corrosion time.
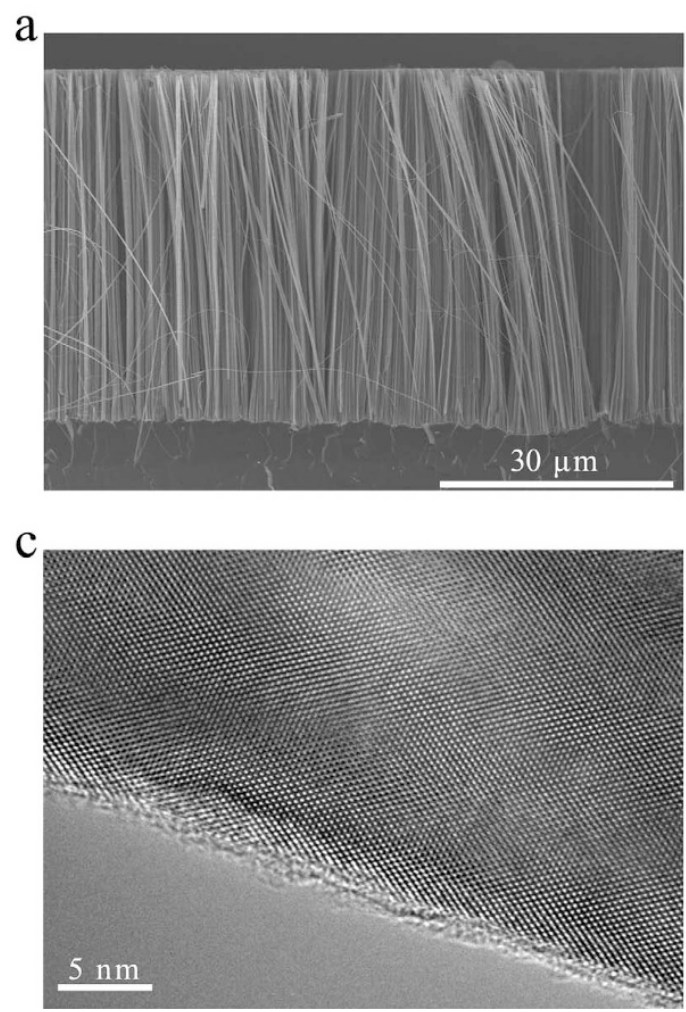

High-resolution transmission electron microscopic (TEM) images in Figure $2 \mathrm{c}$ and $2 \mathrm{~d}$ reveal the single crystalline nature of SiNWs in Figure $2 \mathrm{a}$ and $2 \mathrm{~b}$, respectively. The images show that the axial orientations of the nanowires are along the [100] directions, indicating the preferential anisotropic etching of $\langle 100\rangle$ silicon. HR-TEM images also reveal that the surface of SiNWs from lowdoped p-type $1-10 \Omega \cdot \mathrm{cm} \mathrm{Si}(100)$ wafer is relatively smooth, while that from highly-doped p-type $0.001-0.008 \Omega \cdot \mathrm{cm} \mathrm{Si}(100)$ wafer is extremely mesoporous (Fig. S2). Note that the geometries of etched Si nanostructures are dependent on the pattern or shape of noble metal catalysts, therefore a variety of Si nanostructures with distinct features could be fabricated by precisely predefining the patterns of noble metal catalysts. Fig. S3 shows the typical SEM image of regular silicon nanoholes with controlled geometry and density.

We evaluate the substrate-enhanced MCEE of silicon by galvanic current measurements, which were performed in a home-made electrochemical cell. The silicon area exposed to aqueous HF solution is $1.0 \mathrm{~cm}^{2}$ and the $\mathrm{Si} / \mathrm{Graphite}$ surface area ratio is set to $1: 200$. Figure 3 shows the galvanic current densities as a function of time for silicon and graphite coupled together through an external wire. The galvanic current rises sharply as the circuit is short-circuited and then levels off with time. The initial abrupt drop in corrosion current is due to the limited solubility of oxygen in aqueous HF solution. In the presence of AgNPs, the measured current density of the p-Si/Graphite galvanic cell is considerably larger than that of $n-S i / G r a p h i t e ~ g a l-$ vanic cell in the presence of AgNPs (Fig. 3a), and nearly two orders of magnitude greater than that of $\mathrm{p}$-Si/Graphite galvanic cell in the absence of AgNPs (Fig. 3b). These results are well consistent with our SEM observations. Due to direct contact of noble metal catalysts with silicon in aqueous HF solution, the microscopic Si/AgNPs galvanic cells do not contribute to the measured corrosion current, which originates from the macroscopic Si/Graphite galvanic cell. Assuming a constant corrosion rate, the corrosion current density
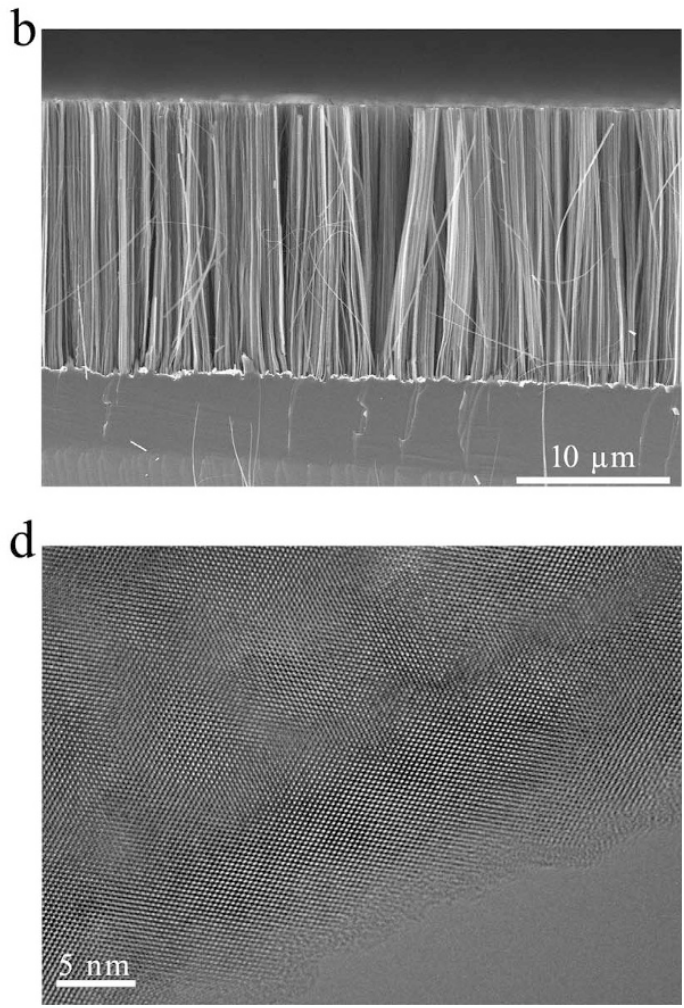

Figure $2 \mid$ SEM and TEM images of SiNWs prepared by graphite substrate-enhanced MCEE of silicon in aerated aqueous HF solution. (a), Crosssectional SEM image of SiNW array prepared on 1-10 $\Omega \cdot \mathrm{cm}$ p-Si(100) wafer. (b), Cross-sectional SEM image of SiNW array prepared on 2-3 $\Omega \cdot \mathrm{cm}$ $\mathrm{Si}(100)$ wafer. (c), High-resolution TEM image of a Si nanowire from 1-10 $\Omega \cdot \mathrm{cm}$ p-Si(100) wafer. (d), High-resolution TEM image of a Si nanowire from $2-3 \Omega \cdot \mathrm{cm} \mathrm{n}-\mathrm{Si}(100)$ wafer. 
a

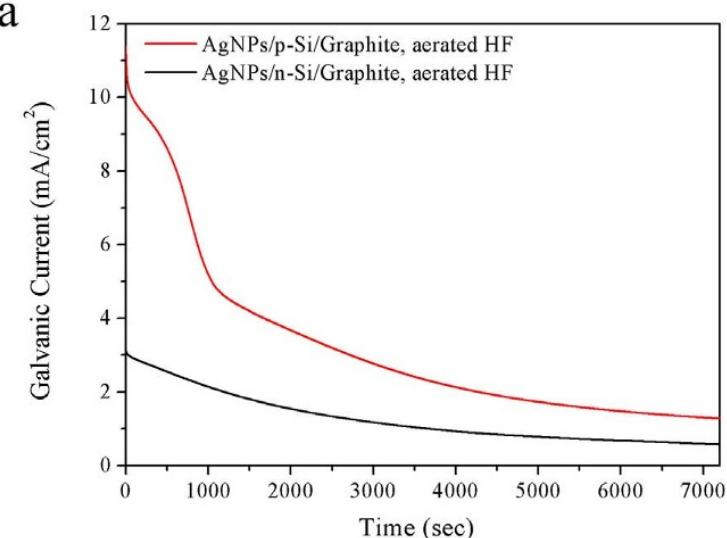

b

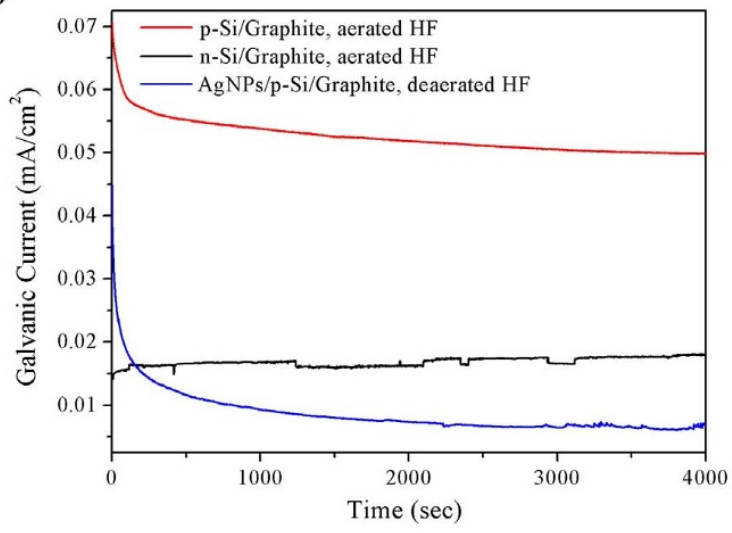

Figure 3 Time dependence of galvanic current for Si and graphite coupled together in aerated aqueous HF solution. (a), AgNPs/p-Si/ Graphite and AgNPs/n-Si/Graphite galvanic cells in aerated aqueous HF solution. (b), p-Si/Graphite and n-Si/Graphite galvanic cells in aerated HF electrolyte; AgNPs/p-Si/Graphite galvanic cells in de-aerated aqueous HF solution. from microscopic Si/AgNPs galvanic cells reaches $1.3 \mathrm{~mA} / \mathrm{cm}^{2}$ using Faraday's law based on the mass loss of silicon and galvanic currenttime curve measured for macroscopic p-Si/Graphite galvanic cell in the presence of AgNPs. The role of dissolved oxygen in aqueous HF solution was investigated by conducting corrosion experiment in deaerated aqueous HF solution bubbling with high-purity nitrogen gas. In the presence of AgNPs, the steady-state current density of the $\mathrm{p}-\mathrm{Si} /$ Graphite galvanic cell in de-aerated aqueous HF solution was $0.007 \mathrm{~mA} / \mathrm{cm}^{2}$ (Fig. 3b), which is three orders of magnitude lower than that of a similar cell in aerated aqueous HF solution, showing silicon corrosion is completely governed by oxygen reduction reaction.

Noble metal substrate-enhanced MCEE of silicon in aerated aqueous HF solution. We further investigated noble metals (Ag, $\mathrm{Au}$, and $\mathrm{Pt}$ ) substrate-enhanced MCEE of silicon in aerated aqueous HF solution for SiNWs fabrication under the same conditions. Figure $4 \mathrm{a}-\mathrm{c}$ show the typical cross-sectional SEM images of SiNW arrays prepared on p-type $1-10 \Omega \cdot \mathrm{cm} \mathrm{Si}(100)$ wafers placed on $\mathrm{Ag}, \mathrm{Au}$ and $\mathrm{Pt}$ substrates, respectively. The etching time is 2 hours. These SEM images clearly reveal that the length of produced SiNWs or the etching rate strongly depends on the type of used noble metal substrates and increases in the order of $\mathrm{Ag}, \mathrm{Au}$, and $\mathrm{Pt}$, indicating these metal substrates show quite different catalytic activity in the cathodic oxygen reduction reaction. To investigate catalytic activities of noble metal substrates in oxygen reduction, we carry out galvanic corrosion experiments by measuring the galvanic current of noble metal substrates coupled with silicon substrates in aerated aqueous HF solution. Figure $4 \mathrm{~d}$ shows the measured galvanic current densities as a function of time for silicon substrates coupled with $\mathrm{Ag}, \mathrm{Au}$, and Pt substrates. It clearly reveals that the galvanic current increases in the same order of $\mathrm{Ag}, \mathrm{Au}$, and $\mathrm{Pt}$, which is well consistent with SEM observations. These results imply that catalytic activities of the cathodes considerably affect oxygen reduction and accompanied silicon etching rates in aerated aqueous HF solution.

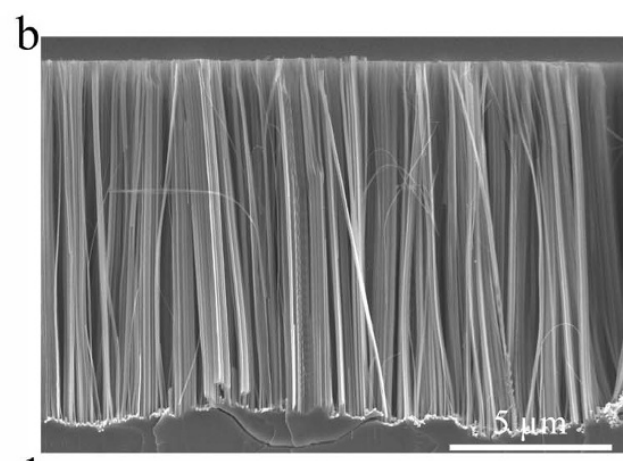

$\mathrm{d}$
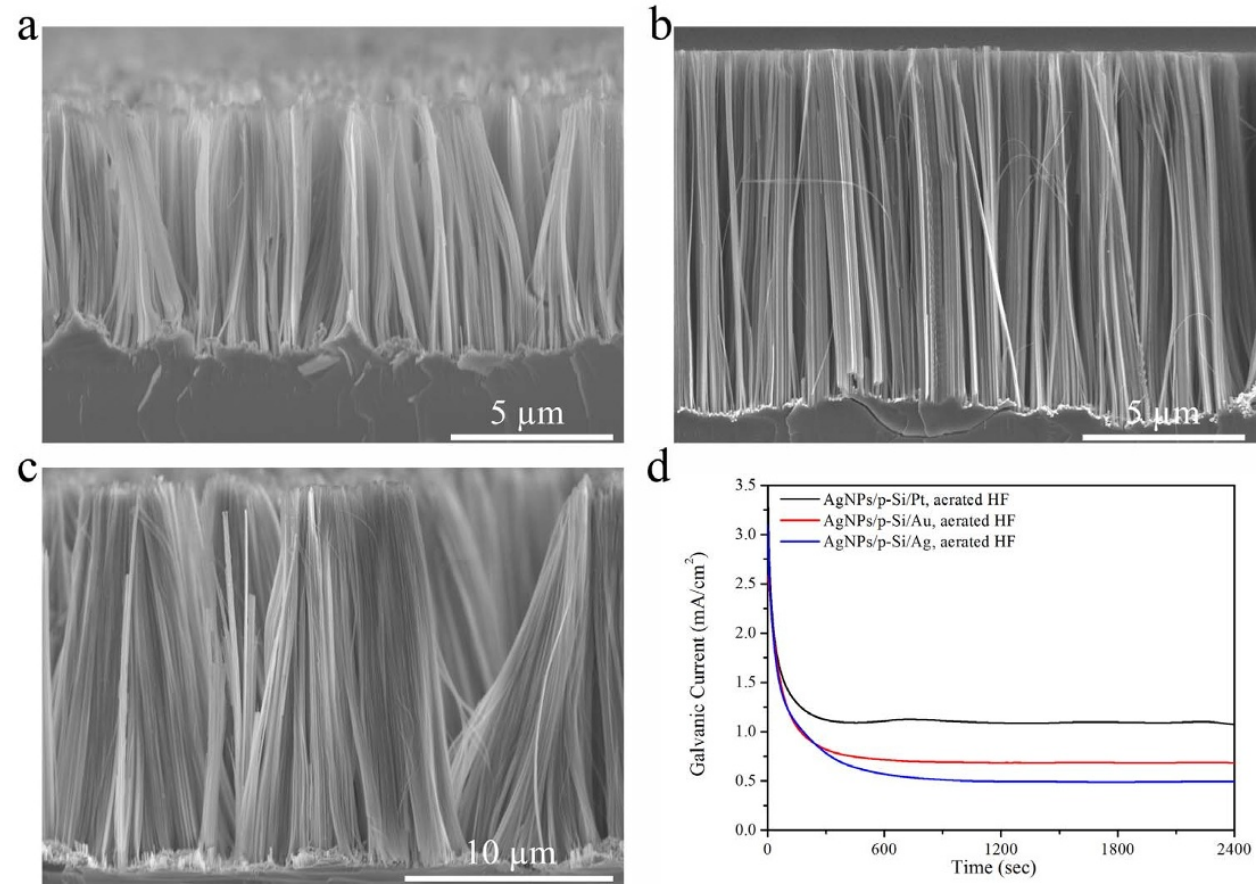

Figure $4 \mid$ Effects of the noble metal substrate types on the corrosion rates of silicon wafers in aerated aqueous HF solution. (a), SEM image of SiNW array prepared by Ag substrate-enhanced MCEE of silicon. (b), SEM image of SiNW array prepared by Au substrate-enhanced MCEE of silicon. (c), SEM image of SiNW array prepared by Pt substrate-enhanced MCEE of silicon. (d), Time dependence of galvanic current for Si coupled with different noble metal substrates in aerated aqueous HF solution. All wafers used are $1-10 \Omega \cdot \mathrm{cm}$ p-Si(100) wafers. 
a

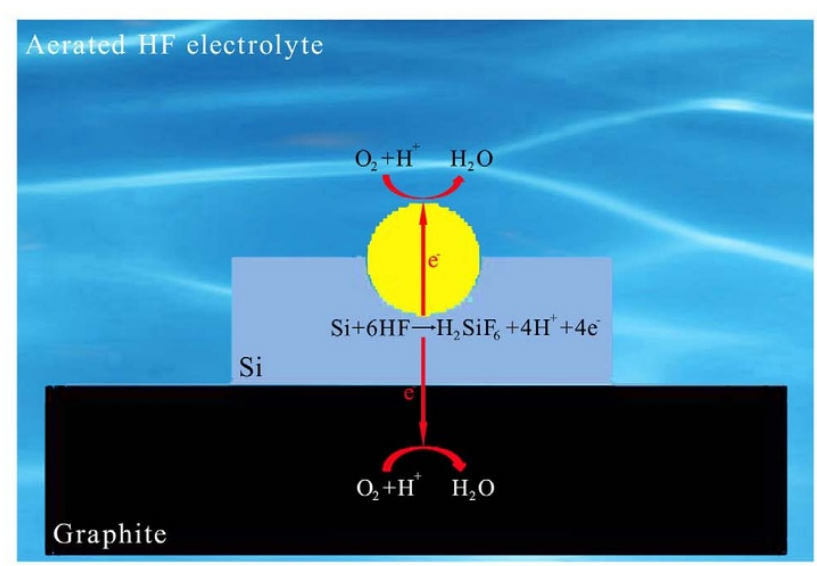

b

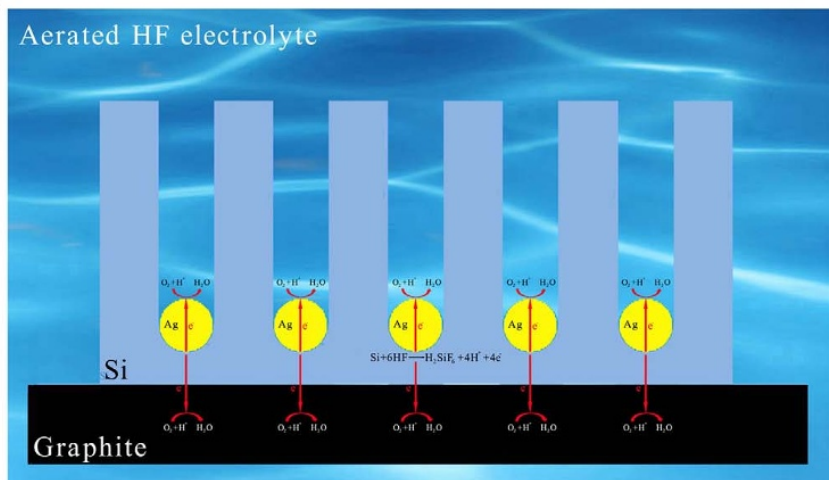

Figure 5 | Schematic illustration of substrate-enhanced MCEE of silicon using dissolved oxygen in aqueous HF solution as an oxidant. (a), Rapid galvanic corrosion of silicon takes place when oxygen is reduced on both noble metal catalysts and graphite, generating electrical current flowing through the Si/metal interface within propagating pits. (b), Highly-

localized selective galvanic corrosion of silicon leads to formation of threedimensional silicon nanostructures with considerable depth.

\section{Discussion}

The above results demonstrate that the present substrate-enhanced MCEE approach is a very simple method for preparing SiNW arrays without needing the common toxic oxidizing agents such as silver nitrate and hydrogen peroxide. In the absence of graphite or noble metal substrates, only shallow pits, as shown in Fig. S4, are produced on $\mathrm{Si}$ surface. Additionally, since only thin porous silicon is produced in the absence of noble metal catalysts, we conclude that the corrosion rate of silicon in the presence of both graphite and noble metal catalysts is much faster than that when either one of them is used alone, revealing the strong synergistic enhancing effect of graphite and noble metal for galvanic corrosion of silicon. An electrochemical model of the synergistic galvanic corrosion process is proposed as schematically illustrated in Fig. 5a. Due to the electrode potential difference, a macroscopic galvanic cell between $\mathrm{Si}$ and graphite, accompanying by microscopic galvanic cells between $\mathrm{Si}$ and noble metal catalysts, is constructed when Si wafer coated with noble metal catalysts are electrically coupled to graphite substrate in aerated aqueous HF solution. While random and stochastic microscopic galvanic cells may exist on $\mathrm{Si}$ surface ${ }^{38}$, they contribute negligibly to silicon corrosion in aerated aqueous HF solution. The anodic and cathodic half-cell reactions for the substrate-enhanced MCEE of silicon are described as follows:

$$
\text { Anodic (Silicon) }: \mathrm{Si}+6 \mathrm{HF} \rightarrow \mathrm{H}_{2} \mathrm{SiF}_{6}+4 \mathrm{H}^{+}+4 e^{-}
$$

Cathodic (Graphite and Metal) : $\mathrm{O}_{2}+4 \mathrm{H}^{+}+4 e^{-} \rightarrow 2 \mathrm{H}_{2} \mathrm{O}$
Briefly, the dissolved oxygen is reduced on both surfaces of graphite and noble metal catalysts, while silicon is oxidized by releasing electrons. Due to enhanced cathodic oxygen reduction, the anodic silicon dissolution reaction is simultaneously accelerated. As current lines always follow the minimal ohmic resistance, thus the electrical current flowing through the silicon/metal interface within the propagating pits would be very intense, resulting in highly selective pitting corrosion of silicon underneath the noble metal catalysts, and consequential formation of silicon nanostructures with considerable depth (Fig. 5b). We suggest that the macroscopic galvanic cell between $\mathrm{Si}$ and graphite in combination with the microscopic galvanic cells between $\mathrm{Si}$ and noble metal catalysts contribute to the intense corrosion current, which is also significantly affected by the charge carrier characteristics of Si substrates. Consequently, the corrosion rate of p-type $\mathrm{Si}$ is faster due to the abundant holes in $\mathrm{p}$-Si, while that of n-type $\mathrm{Si}$ is slower due to the limited supply of holes.

In conclusion, we demonstrate for the first time the simple, continuous-flow mass production of SiNWs via substrate-enhanced MCEE of silicon using dissolved oxygen in aqueous HF solution as a green oxidant. The distinct advantages of this novel approach such as simplicity, scalability and flexibility would make it an attractive alternative to conventional MCEE methods. Since galvanic corrosion occurs in all semiconductor-metal and metal-metal systems, we suggest that such galvanic corrosion processes may be generally adopted to fabricate other semiconducting and metallic nanostructures.

\section{Methods}

Fabrication of silicon nanowires. SiNWs can be prepared on different starting $\mathrm{Si}$ materials, including single-crystalline silicon wafer, multi-crystalline silicon wafer, thin silicon film, and silicon particles, by means of substrate-enhanced MCEE of silicon in a continuous production line consisting of graphite or noble metal (Ag, Au, and $\mathrm{Pt}$ ) substrates immersed in aerated aqueous HF solution. To prepare high-aspect ratio SiNWs, Si materials must be pre-coated with thin noble metal (Ag, Au) particles before being placed onto graphite or noble metal substrates immersed in aerated aqueous HF solution. Only porous silicon was prepared from clean Si materials placed on a graphite substrate immersed in aerated aqueous HF electrolyte (10\% in weight) for 10-300 minutes. All experiments were carried out at room temperature. All chemicals were purchased from Sigma-Aldrich.

Structural and electrochemical characterization. The morphologies and microstructures of silicon nanostructures were investigated using a field-emission scanning electron microscope (SEM, HITACHI S-4800) and a transmission electron microscope (TEM, JEOL JEM-2100). The galvanic corrosion current was measured in a Teflon-made electrochemical cell. The de-aerated aqueous HF electrolyte was prepared by purging high-purity $\mathrm{N}_{2}$ through the solution for 1 hour. The electrochemical measurements were performed using a Zennium electrochemical workstation (IM6, Zahner, Germany) with silicon as the working electrode and a graphite rod as the counter electrode. Ohmic contact was made by pasting high-purity In-Ga alloy onto the rear side of the Si wafer with $1 \mathrm{~cm}^{2}$ of the front surface area exposed to aqueous HF solution.

1. Morales, A. M. \& Lieber, C. M. A laser ablation method for the synthesis of crystalline semiconductor nanowires. Science 279, 208-211 (1998).

2. Ma, D. D. D., Lee, C. S., Au, F. C. K., Tong, S. Y. \& Lee, S. T. Small diameter silicon nanowire surface. Science 299, 1874-1877 (2003).

3. Hannon, J. B., Kodambaka, S., Ross, F. M. \& Tromp, R. M. The influence of the surface migration of gold on the growth of silicon nanowires. Nature 440, 69-71 (2006).

4. Wang, Y., Schmidt, V., Senz, S. \& Gösele, U. Epitaxial growth of silicon nanowires using an aluminum catalyst. Nat. Nanotech. 1, 186-189 (2006)

5. Hochbaum, A. I., Fan, R., He, R. \& Yang, P. Controlled growth of Si nanowire arrays for device integration. Nano Lett. 5, 457-60 (2005).

6. Renard, V. T. et al. Catalyst preparation for CMOS-compatible silicon nanowire synthesis. Nat. Nanotech. 4, 654-657 (2009).

7. Schmidt, V., Wittemann, J. V., Senz, S. \& Gösele, U. Silicon nanowires: a review on aspects of their growth and their electrical properties. Adv. Mater. 21, 2681-2702 (2009).

8. Cui, Y. \& Lieber, C. M. Functional nanoscale electronic devices assembled using silicon nanowire building blocks. Science 291, 851-853 (2001).

9. Hochbaum, A. I. et al. Enhanced thermoelectric performance of rough silicon nanowires. Nature 451, 163-167 (2008).

10. Peng, K. Q., Wang, X., Li, L., Hu, Y. \& Lee, S. T. Silicon nanowires for advanced energy conversion and storage. Nano Today 8, 75-97 (2013). 
11. Chan, C. K. et al. High-performance lithium battery anodes using silicon nanowires. Nat. Nanotech. 3, 31-35 (2008).

12. Tian, B. et al. Coaxial silicon nanowires as solar cells and nanoelectronic power sources. Nature 449, 885-890 (2007).

13. Zheng, G., Patolsky, F., Cui, Y., Wang, W. U. \& Lieber, C. M. Multiplexed electrical detection of cancer markers with nanowire sensor arrays. Nat. Biotechnol. 23, 1294-1301 (2005).

14. Kelzenberg, M. D. et al. Enhanced absorption and carrier collection in Si wire arrays for photovoltaic applications. Nat. Mater. 9, 239-244 (2010).

15. Boettcher, S. W. et al. Energy-conversion properties of vapor-liquid-solid-grown silicon wire-array photocathodes. Science 327, 185-187 (2010).

16. Shen, X., Sun, B., Liu, D. \& Lee, S. T. Hybrid heterojunction solar cell based on organic-inorganic silicon nanowire array architecture. J. Am. Chem. Soc. 133, 19408-19415 (2011).

17. Peng, K. Q., Yan, Y. J., Gao, S. P. \& Zhu, J. Synthesis of large-area silicon nanowire arrays via self-assembling nanoelectrochemistry. Adv. Mater. 14, 1164-1167 (2002)

18. Li, X. \& Bohn, P. W. Metal-assisted chemical etching in $\mathrm{HF} / \mathrm{H}_{2} \mathrm{O}_{2}$ produces porous silicon. Appl. Phys. Lett. 77, 2572-2574 (2000).

19. Peng, K. Q. et al. Fabrication of single-crystalline silicon nanowires by scratching a silicon surface with catalytic metal particles. Adv. Funct. Mater. 16, 387-394. (2006)

20. Huang, Z., Fang, H. \& Zhu, J. Fabrication of silicon nanowire arrays with controlled diameter, length, and density. Adv. Mater. 19, 744-748 (2007).

21. Peng, K. Q., Lu, A. J., Zhang, R. Q. \& Lee, S. T. Motility of metal nanoparticles in silicon and induced anisotropic silicon etching. Adv. Funct. Mater. 18, 3026-3035 (2008)

22. Choi, W. K. et al. Synthesis of silicon nanowires and nanofin arrays using interference lithography and catalytic etching. Nano Lett. 8, 3799-3802 (2008).

23. Hochbaum, A. I., Gargas, D., Hwang, Y. J. \& Yang, P. D. Single crystalline mesoporous silicon nanowires. Nano Lett. 9, 3550-3554 (2009).

24. Peng, K. Q. et al. Uniform, axial-orientation alignment of one-dimensional singlecrystal silicon nanostructure arrays. Angew. Chem. Int. Ed. 44, 2737-2740 (2005).

25 . Huang, Z. et al. Ordered arrays of vertically aligned [110] silicon nanowires by suppressing the crystallographically preferred etching directions. Nano Lett. 9 , 2519-2525 (2009).

26. Chen, H., Wang, H., Zhang, X. H., Lee, C. S. \& Lee, S. T. Wafer-scale synthesis of single-crystal zigzag silicon nanowire arrays with controlled turning angles. Nano Lett. 10, 864-868 (2010)

27. Huang, Z., Geyer, N., Werner, P., Boor, J. \& Gösele, U. Metal-assisted chemical etching of silicon: A review. Adv. Mater. 23, 285-308 (2011).

28. Peng, K. Q. et al. Ordered silicon nanowire arrays via nanosphere lithography and metal-induced etching. Appl. Phys. Lett. 90, 163123 (2007).

29. Weisse, J., Kim, D., Lee, C. \& Zheng, X. Vertical transfer of uniform silicon nanowire arrays via crack formation. Nano Lett. 11, 1300-1305 (2011).

30. Peng, K. Q., Wang, X., Li, L., Wu, X. L. \& Lee, S. T. High-performance silicon nanohole solar cells. J. Am. Chem. Soc. 132, 6872-6873 (2010).
31. Peng, K. Q. et al. Aligned single-crystalline Si nanowire arrays for photovoltaic applications. Small 1, 1062-1067 (2005).

32. Garnett, E. C. \& Yang, P. D. Silicon nanowire radial p-n junction solar cells. J. Am. Chem. Soc. 130, 9224-9225 (2008).

33. Sivakov, V. et al. Silicon nanowire-based solar cells on glass: synthesis, optical properties, and cell parameters. Nano Lett. 9, 1549-1554 (2009).

34. Huang, R., Fan, X., Shen, W. \& Zhu, J. Carbon-coated silicon nanowire array films for high-performance lithium-ion battery anodes. Appl. Phys. Lett. 95, 133119 (2009).

35. Peng, K. Q., Wang, X. \& Lee, S. T. Gas sensing properties of single crystalline porous silicon nanowires. Appl. Phys. Lett. 95, 243112 (2009).

36. Wang, X. T., Shi, W. S., She, G. W. \& Mu, L. X. Using Si and Ge Nanostructures as substrates for surface-enhanced Raman scattering based on photoinduced charge transfer mechanism. J. Am. Chem. Soc. 133, 16518-16523 (2011).

37. Chen, L. et al. Aptamer-mediated efficient capture and release of T llymphocytes on nanostructured surfaces. Adv. Mater. 23, 4376-4380 (2011).

38. Turner, D. R. On the mechanism of chemically etching germanium and silicon. J. Electrochem. Soc. 107, 810-816 (1960).

\section{Acknowledgments}

We acknowledge financial support from National Natural Science Foundation of China (51072025), National Basic Research Program of China (2012CB932400), Beijing Natural Science Foundation (2112021), The Fundamental Research Funds of the Central Universities (2012LZD02), and Fok Ying Tung Education Foundation (121047).

\section{Author contributions}

Y.H. and K.Q.P. designed the experiments. Y.H., L.L., Z.Q. and X.L.W. performed the fabrication experiments and electrochemical measurements. X.H. and X.M.M. performed TEM characterization. K.Q.P. supervised the project, performed data analysis and wrote the paper. S.T.L. discussed the results, performed data analysis and contributed to manuscript writing. All the authors reviewed and commented on the manuscript.

\section{Additional information}

Supplementary information accompanies this paper at http://www.nature.com/ scientificreports

Competing financial interests: The authors declare no competing financial interests.

How to cite this article: $\mathrm{Hu}$, Y. et al. Continuous-flow Mass Production of Silicon Nanowires via Substrate-Enhanced Metal-Catalyzed Electroless Etching of Silicon with Dissolved Oxygen as an Oxidant. Sci. Rep. 4, 3667; DOI:10.1038/srep03667 (2014).

This work is licensed under a Creative Commons AttributionNonCommercial-NoDerivs 3.0 Unported license. To view a copy of this license, visit http://creativecommons.org/licenses/by-nc-nd/3.0 